\title{
Sistemi di propulsione elettrotermica $(*)$
}

\author{
R. MoNTI $(* *)$ \\ Ricevuto il 18 Maggio 1963
}

Russsuxto. - Si pongono in risalto le limitazioni della propulsione chimica rispetto a quella termica non convenzionale e si esaminano i problemi e le applicazioni dei sistemi elettrotermici. Si illustrano alcune proposte per migliorare il processo di riassociazione all'interno dell'ugello in presenza di fenomeni di non-equilibrio chimico.

Summar. - The problems related to the construction and to the applications of the electrothermal propulsive devices are pointed out. The nozzle behavior is examined when chemical non-equilibrimm phenomena are present because of the high temperature and the high velocity of the gas propellant.

With reference to a linearized analysis wich yelds some information about the design parameters to be considered in the present case, some proposal, for increasing the so called non-equilibrium efficiency, are considered either suitably shaping the nozzle contonr or trying to influence the chemical kinetics of the dissociation reaction during the propellant expansion inside the nozzle.

The mentioned proposals will be considered in details in the next future.

\section{1. - INTRODUZIONE.}

Sistemi propulsivi termici sono quelli che convertono energia termica contenuta in un propellente ad alta temperatura, a mezzo di una espansione in un ugello, in energia cinetica. L'ugello e quindi il sistema che converte energia disordinata delle particelle (energia termica) in energia ordinata (energia cinetica del getto).

$\left(^{*}\right)$ Nota presentata al $2^{\circ}$ Congresso Internazionale Teconico Scientifico dello Spazio. Roma, 19-23 Gingno 1962.

$\left(^{* *}\right)$ Istituto di Aeronantica. Lniversita di Napoli. 
La velocita di scarico di un ugello, se il processo all'interno di esso e adiabatico, puo immediatamente ricavarsi dalla conoscenza delle entalpie totali per unita di massa (sensibili pii quelle di riferimento) all'ingresso dell'ugello $\left(h_{i}\right)$ e all'uscita da esso $\left(h_{n}\right)$ dalla:

$$
V_{u}=\sqrt{2} \sqrt{h_{i}-h_{u}} .
$$

La [1] esprime un bilancio energetico egguagliando la diminuzione di entalpia alla energia cinetica del getto nel caso che $\mathbf{T}_{i}=0$.

Introducendo i rendimenti $\varepsilon_{\text {tch }} \varepsilon_{\text {ad }}$ definiti in seguito, nella (1), e indicando con $H_{i}$ la entalpia per $\mathrm{Kg}$ mole (Cal/Kg mole), con $m$ il peso molecolare del gas e con $I_{s p}$ l'impulso specifico (secondi), si ha:

$$
I_{s p}=\frac{V_{u}}{q}=I \sqrt{\varepsilon_{\mathrm{Lchl}} \varepsilon_{\mathrm{ad}}} \mid \frac{H_{i}}{m} .
$$

La [2] sintetizza i limiti e le possibilità dei sistemi propulsivi termici. Infatti l'impulso specifico che, pur non essendo l'unico parametro da prendere in esame nei sistemi di propulsione spaziale, e il parametro di maggiore importanza per missioni spaziali particolarmente lontane ( $\left.{ }^{*}\right)$, può essere incrementato, rispetto ai sistemi convenzionali, soltanto se:

- Si aumenta il valore di $H_{i}$ cioe della energia posseduta dalle singole particelle all'ingresso dell'ugello; tale energia e, per ogni propellente, funzione crescente della temperatura.

- Si diminuisce $m$ cioe, a pari $H_{i}$, si aumenta l'energia per unita di peso di fluido.

- Si migliorano valori del prodotto $\varepsilon_{\text {tch }} \varepsilon_{\text {ad }}$.

Il processo secondo cui viene incrementata la temperatura del propellente è diverso a seconda che si tratta di:

1) razzi chimici nei quali avviene un processo di combustione che converte l'energia chimica (energia potenziale inerente alla struttura

(*) Il valore di $I s p$ che ottimizza la massa totale del veicolo spaziale per un tipo di sistema propulsivo e per una assegnata missione spaziale, individuata normalmente dal valore dell'incremento della velocita e dal carico utile, cresce con la lontananza della missione stessa. Per i sistemi chimici e per un dato propellente si raggiunge un compromesso tra incrementi di Isp, e la conseguente diminuzione del peso del propellente, e incremento del peso strutturale al crescere del rapporto delle pressioni. Per i sistemi non convenzionali il compromesso e raggiunto tra peso del propellente necessario (che diminuisce con $I s p$ ) e peso dell'apparato sorgente-convertitore-greneratore di spinta (la cui potenza e peso cresce con $I s p$ ). 
molecolare del propellente) in energia termica (energia media competente ai gradi di liberta interni, traslatori, rotat ori, vibratori) e in altre forme di (energia (dissociazione, ionizzazione).

2) Razzi nucleari, solari o elettrotermici per i quali l'energia viene reduta al propellente da una sorgente esterna a mezzo di trasmissione di calore o direttamente (sistema solare o nucleare) o tramite la conversione di essa energia in energia elettrica; in questi ultimi tipi vi e ovviamente necessita di apparati di conversione dellenergia, messa a disposizione dalla sorgente, in energia elettrica e di particolari sistemi che conrertono l'energia elettrica, in energia termica da trasmettere al propellente (archi elettrici, resistenze ohmiche, ecr.).

Per i razzi chimici il valore di $H_{i}$ e limitato dal contenuto energetico dei carburanti (per rarburanti avanzati del tipo $H_{2}-\mathrm{O}_{2}, H_{i} \simeq 25 \mathrm{Cal} / \mathrm{gm}$ mole) mentre per i sistemi non convenzionali, in particolare gli elettrotermici, il valore di $H_{i}$ e teoricamente illimitato nel senso che, se non intervenissero difficolta teconologiche e di disponibilita di energia, si potrebbe immettere una quantita di energia, per particella, grande a piacere: in pratica, per esempio, per $H_{2}$ il valore limite tecnologico (corrispondente ad una temperatura di $15.000 \circ \mathrm{S})$ e di $H_{t} \simeq 200 \mathrm{Cal} / \mathrm{urg}$ mole. Pertanto solo con i sistemi di propulsione aranzata, che utilizzano il principio di immettere nel propellente energia messa a disposizione da nn'altra sorgente anziché sfruttare solo quella rhimica contenuta nel propellente stesso, si possono realizzare grandi $I s p$ e quindi compiere missioni negate ai propellenti chimici. Per i sistemi chimici, inoltre, vi è la ulteriore limitazione del valore di $m$, doruta alla presenza dell'ossidante che ha un peso molecolare molto grande. Questa e forse la ragione principale che ha spinto verso nuove soluzioni; infatti, anche con un valore di $H_{\imath}$ pari a quello che si ha per combustione $\mathrm{H}_{0}-\mathrm{O}_{2}$ con rapporto corrispondente all'impuslo specifico massimo $\left(n_{H_{2}} / n_{O_{2}} \simeq 4\right)$ un sistema elettrotermico, con propellenter $H_{\text {, }}$, raggiunge un impulso specifico circa 2,5 volte maggiore del sistema chimico.

\section{2. - SIILUPPO .ITTUAI.E DEI MOTORI ELETTRO'TERMICI.}

Tra i sistemi temici non conrenzionali (non chimici) il sistema di propulsione alettrotermica riceve noterolissima attenzione ed e quello in fase decisamente piu aranzata anche perche il suo componente prin(cipale (arco elettrico) è stato ed e attualmente sviluppato anche per applicazioni non propulsive (plasma-jet, tumnel a vento ad areo, ecc.) che 
arlotano un arco elettrico dalle earatteristiche molto simili a quello di un arco-jet.

Innanzitutto vale la pena richiamare quali sono le potenzialita di un sistema propulsivo elettrotemico con particolare riferimento al possibile impiego spaziale che si sta concretizzando con la messa a punto del progetto SNAP 8 e di altri reattori nucleari. Come e noto il reattore nucleare SNAP 8, generatore di energia elettria per impieghi spaziali, produce una potenza elettrica di $30060 \mathrm{~K} W$ a secondo che si prevedono uno o due gruppi di turbogenelatori.

Molti studi e proposte di missioni spaziali sono state fatte prevedendo l'impiego dello SNAP 8 con sistemi di propulsione elettrotermica, a ioni ed a plasma, tra essi il piu immediato sembra sia proprio un sistema ad arco elettrico. Per tale sistema le missioni possibili sono molte e si cita qui solo quella attualissima del lancio, nel piano equatoriale terrestre, di un satellite per comunicazioni che ruoti con la stessa velocita angolare della terra in modo da risultare fisso rispetto ad un osservatore terrestre.

In linea di massima i vantagrgi dell'uso di un razzo ad arco rispetto ad un razzo chimico sono:

1) Possibilita di portare carichi maggiori anche se in tempi piu lunghi (data la piccolezza del rapporto spinta/peso);

2) Ottima possibilità della regolazione della spinta;

3) Disponibilita di una grande potenza elettrica ma volta portata a compimento la missione.

Queste caratteristiche renclono particolamente adatto questo sistema propulsivo per la missione sopracitata. Si pensa $\left(^{6}\right)$ jer esempio, di variazione delle dimensioni, sostitnzione delle parti consunte ed inquiimmettere un razzo (di circal $4000 \mathrm{~kg}$ ) inizialmente su un'orbita di circa $500 \mathrm{~km}$, a mezzo di un booster Atlas e successivamente, a mezzo di un arco-jet alimentato dalla SNAP $8(0, \breve{o} \mathrm{~kg}$ di spinta con $I s p-1100$ sec.) di portare nell'orbita prevista (circa $36.000 \mathrm{~km}$ ), con una traiettoria a spirale, il carico utile di circa $900 \mathrm{~kg}$ piu la centrale elettrica. Questo per mettere in risalto lattuabilita del problema e la sua pratica attuazione.

Un arco-jet e composto semplicemente da due elettrodi di cui uno (l'anodo) a a forma di ugello e l'altro a forma di una spina conica (Vedi Fig. 1); normalmente il propellente entra nella zona dell'arco tangenzialmente in modo da provocare una rotazione dell'arco, in tal modo evi- 
tando punti caldi a le conseguenti forti erosioni, e provvedendo a rendere uniforme la temperatura e la composizione del gas all'ingresso dell'ugello. I problemi principali di un sistema propulsivo ad arco sono:

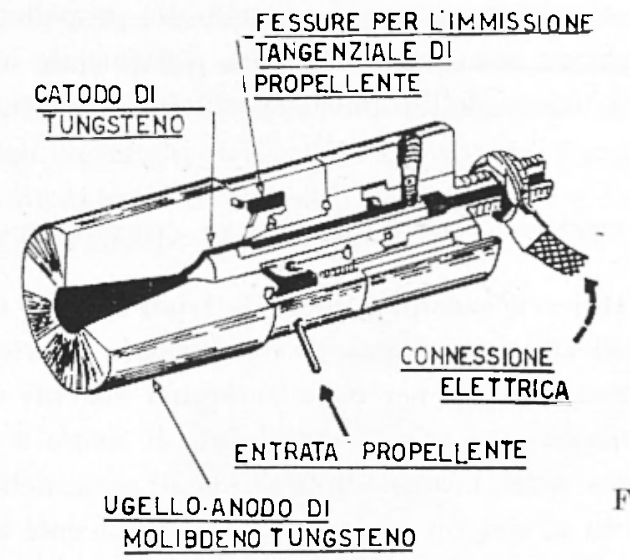

Fig. 1

- consumo degrli elettrodi;

- inquinamento del flusso;

- raftreddamento:

- rendimento di conversione;

- uniformizzazione del propellente a monte dell'ugello;

- scelta del propellente;

- tipo di potenza elettrica da impiegarsi;

- avviamento dell'arco;

- progetto dell'ugello.

Questi per citare solo i problemi piu importanti o, comunque, quelli piì caratteristici di questo sistema: evidentemente, accanto ai problemi citati, esistono quelli comuni a tutti i sistemi propulsivi avanzati che prevedono l'impiego di un reattore nucleare: peso, dimensioni, schermaggio, sicurezza di funzionamento, regolazione dei valri componenti ere.

Si discutono brevemente i vari problemi elencati e si prenclera poi in asame più dettagliato la progettazione dell'ugello ed i problemi relativi.

\section{3. - Consumo degli Elettrodi - Ixquinamexto.}

La realizzazione di una durata degli elettrodi molto lunga e stato uno dei problemi piu spinosi della evoluzione degli archi elettrici; se per applicazioni terrestri, possono essere tollerate forti erosioni, nello spazio, invece, avere una dumata lunga e una "conditio sine qua non " pel le ovvie complicazioni di alimentazione degli elettrodi che si comsumano. 
namento del getto con i relativi peggioramenti delle caratteristiche propulsive.

Infatti, dato l'elevatissimo peso molecolare delle sostanze metalliche costituenti gli elettrodi, paragonato a quello del propellente (che e circa 2 ordini di grandezza minore), anche una percentuale bassa di erosione puo peggiorare il valore dell'impulso specifico del sistema.

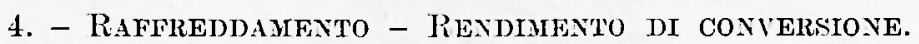

Per poter ridurre il consumo degli elettrodi e poter avere valori non eccessivi delle sollecitazioni termiche e necessario prevedere un raffreddamento. Il raffreddamento per convezione nei sistemi di laboratorio e notevolmente semplificato per la possibilità di avere a disposizione un liquido refrigerante senza nessuna limitazione di peso; nello spazio, invece, bisognera ricorrere ai sistemi gia proposti ed impiegati nei razzi chimici per valori molto alti della trasmissione di calore e cioe sistemi a rigener"azione (a film di liquido o a traspirazione attraverso gli elettrodi).

Il rafireddamento del sistema evidentemente costituisce una perdita per il sistema propulsivo. Si puo definire un rendimento globale di conversione $\left(\varepsilon_{\text {con }}\right)$ come il rapporto tra l'energia elettrica a disposizione $\left(P_{e}=I V\right)$ e quella effettivamente somministrata al fluido $\left.A H_{i} \cdot w\right)$.

$$
\varepsilon_{\mathrm{con}}-\frac{\Delta H_{i}}{\bar{I} \bar{v}} w
$$

dove $w$ è la portata di massa di propellente e $\Delta H_{i}$ l'energia incamerata dal propellente per unita di massa.

Il rendimento $\varepsilon_{\text {con }}$ cosi definito dipendera anche dalle caratteristiche elettriche del sistema. Piu precisamente la corrente elettrica (I) di alimentazione dell'arco dovia circolare anche negli elettrodi e nei circuiti ad essi connessi; questa corrente e l'inevitabile resistenza elettrica degli elettrodi e dei circuiti dara luogo a una potenza dissipata per effetto Joule $I^{2} R_{e}=V_{e} I$ dove $V_{e}$ e la caduta nel circuito (principalmente negli elettrodi). Se si indica con $V_{a}$ la caduta Ohmica attraverso l'arco, tenendo presente che la potenza dissipata nel circuito può ritenersi persa ai fini propulsivi, si potra definire ancora un rendimento elettrico $\left(\varepsilon_{e l}\right)$ come rapporto tra la potenza utilizzabile (a meno di perdite per trasmissione di calore) e quella totale necessaria per far funzionare il sistema ( $I V)$

$$
\varepsilon_{e l}=\frac{P_{a}}{P}=\frac{I V_{a}}{I\left(V_{a}+V_{e}\right)}=\frac{1}{1+\frac{R_{e}}{R_{a}}}
$$


quindi il valore del rapporto $R_{e} R_{a}$ e indicativo del rendimento di funzionamento dell'ar(o). Questo valore dipende, oltre che da altri fattori, anche dalla potenza di gioco perche, all'aumentare della potenza, aumenta la temperatura, il grado di ionizzazione, e quindi la conducibilita del propellente nella zona dell'arco; cio provoca un aumento del rapporto $R_{e} / R_{a}$ e una diminuzione del rendimento $\varepsilon_{e l}$ e quindi di $\varepsilon_{\text {con. }}$ I problemi della durata degli elettrodi, dell'inquinamento del flusso, del raffreddamento e del rendimento di conversione sono legati tra loro e la soluzione migliore e un compromesso tra i diversi fattori.

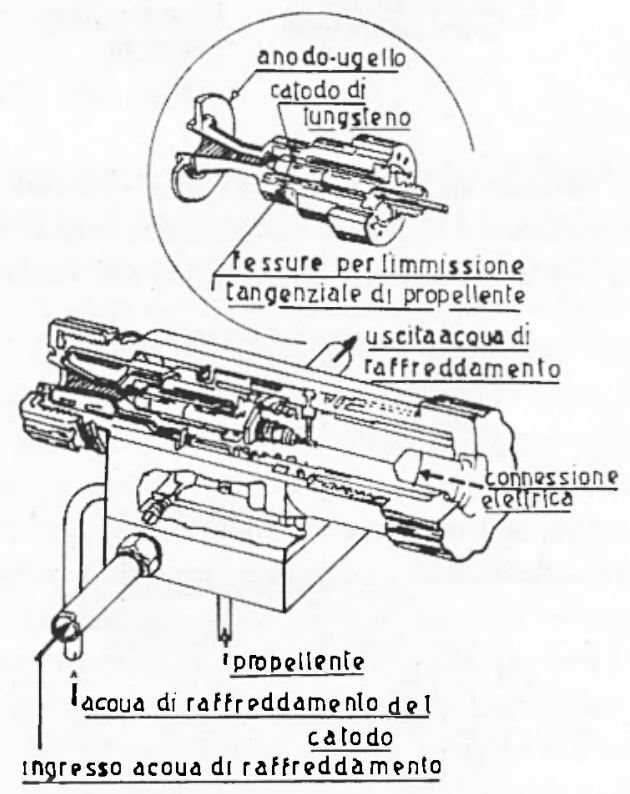

Fig. 2

Se il sistema di raffreddamento prescelto e per radiazione (Vedi Fig. 1) allora cura particolare va posta nella scelta di materiali, altamente refrattari, e nella realizzazione di una temperatura il piu uniforme possibile onde evitare punti caldi, e quindi fusioni o sublimazioni locali e, contemporaneamente, un inquinamento del getto, con conseguente innalzamento del peso molecolare e riduzione di Isp. Una soluzione caratteristica per il raffreddamento ad acqua, per apparati sperimentali da laboratorio, e illustrata in Fig. 2. Il materiale impiegato comunemente per il catodo e il tungsteno e per l'anodo, invece, a seconda del tipo di raffreddamento, rame o tungsteno. 
Un'altra possibile soluzione di alcuni dei problemi elencati e precisamente dell'inquinamento del getto e delle erosioni degli elettrodi prevede un movimento dell'arco elettrico in modo tale che esso non resti fisso

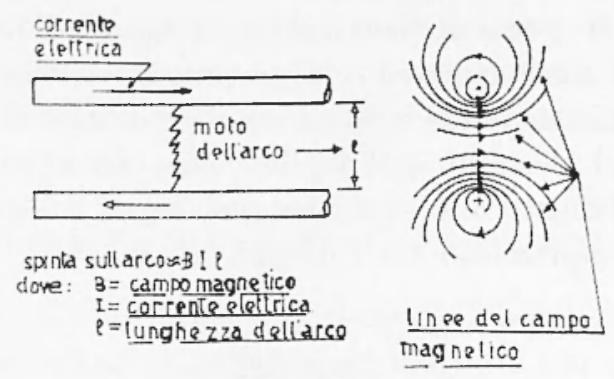

Fig. 3

ma ruoti intorno all'asse di simmetria di due elettrodi concentrici. A questo scopo il propellente e immesso tangenzialmente in modo da provocare una rotazione dell'arco, oppure, sfruttando l'interazione tra la scarica

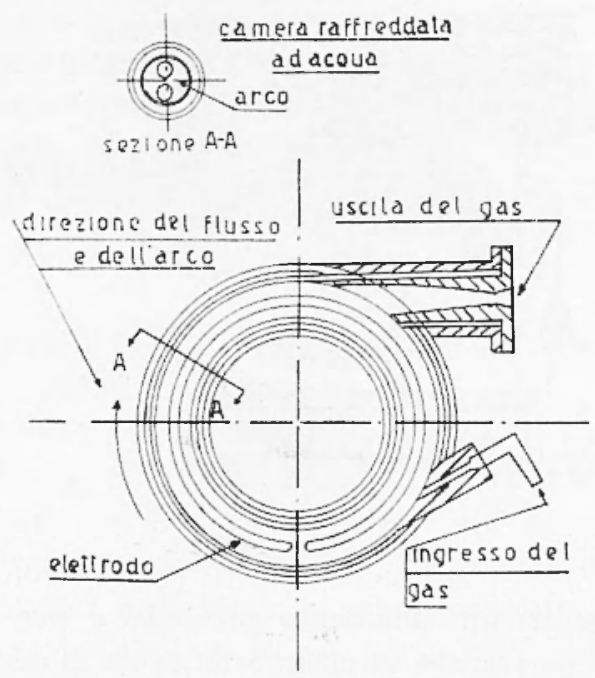

Nigr. 4

elettrica e un campo magnetico, generulmente quello indotto dalla stessa corrente di alimentazione dell'arco (Vedi Fig. 3), si mette in moto l'arco migliorando la uniformita del gas all'ingresso dell'ugello.

La proposta dei metodi accennati è giustificata anche dalla considerazione che, per migliorare il rendimento del trasferimento di energia tra larco elettrico e il fluido, bisogna provocare il massimo numero di colli- 
sioni tra le particelle di fluido, attraverso le quali passa lauco elettrico, a che quindi sono altamente eceitate nei diversi grali di liberta, e le altre che vengono riscaldate proprio in seguito a collisione con le prime. Questo si realizza creando nell'unita di tempo un gran numero di tali particelle eccitate (forti correnti) con un libero cammino medio corto (altal pressione) e interessalndo un forte volume di fluido (noto rapido dell'arco). Il principio e sfruttato nellareo jet illustrato in Fig. 4 nel quale un areo tra due elettrodi concentrici circolari a messo in rotazione, con velocita maggriore della velocita del flusso tangenziale di propellente.

\section{5. - Uxmokmita DeI FILSSO.}

In genere per poter ottenere ma buona uniformita del getto bisognerebbe inmettere il propellente ad alta temperatura in una camera di ristagno nella quale, a mezzo di collisioni, le particelle, provenienti direttamente dalla zona dellareo, possano cedere ad altre che posseggono energia minore, quella parte dellenereia eccestente l'energia media. In questa fase, quindi, parte dell'(2neryia di ionizzazione a di dissociazione delle particelle, interessate dallareo elettrico, viene lecuperata, dal punto di vista propulsivo, perehe ceduta e convertita in energia termica delle particelle circostanti piu fredele. E conveniente poter disporre di una amera di ristagno, le eni dimensioni vamno fissate prendendo in considelazione i tempi caratteristici per gli scambi energetici tra le particelle $\left({ }^{10}\right)$, peso, dimensioni, flusso di energia sotto forma di calore attraverso le pareti. geometria e difficolta tecologiche. Anehe se la turbolenza facilita molto un livellamento eneregetico tuttavia essal da luogo a dissipazioni e pertanto bisogna limitarne l'entita a valori aceettabili: uno dei sistemi é, per esempio, quello gia illustrato in Fig. to che sembra possa diminuire la turbolenza a mezzo di un riscaldamento del gas piid graduale di altri sistemi proposti. Il problema della protezione dell'ugello e di altre parti, sollecitate termicamente, e comme a quello relativo ai razzi ehimici.

\section{6. - Selat Det. propelateste.}

Per quanto riguarda la scelta del propellente la questione e molto dibattuta tra $H_{2}, H_{e}, A_{2}, \mathrm{NH}_{3}$ a bipropellenti (tipo $\mathrm{H}_{2}+\mathrm{L}_{i}$ ). Ognumo di questi propellenti presenta caratteristiche tali da farli prefe- 
rie in applicazioni particolari. I fattori che giocano nella scelta di un propellente sono svariati a precisamente si auspica:

- un basso peso molecolare $m$;

- un propellente non criogenico (qualita in genere in contraddizione con la precedente);

- buone caratteristiche di immagazzinamento, maneggerolezza, non tossicita, alto peso spercifico;

-- bassa reattivita, soprattutto con i materiali costituenti gli elettrodi;

- basso numero di atomi costituenti la molecola. Buone caratteristiche di dissociazione e ionizzazione. Caratteristiche tali da generare un arco elettrico stabile.

Tutti i requisiti elencati sono ovvi, una menzione merita soltanto l'ultimo gruppo. Una molecola con molti atomi oltre a comportare, necessariamente, un forte peso molecolare comporta, ad alte temperature, forti valori dell'energia spesa per la dissociazione del propellente; questa energia e, nella migliore delle ipotesi, recuperata nell'ugello con grado di sfruttabilita minore (l'energia liberata dalle reazioni di riassociazione viene fornita al propellente a pressioni piu basse di quella della camera di ristagno).

In Fig. 5 si riportano le caratteristiche dei piu importanti tipi di propellenti proposti. Si nota come per l'idrogeno è possibile realizzare il massimo $1 s p$, a parita di temperatura di ristagno, rispetto ad altri propellenti, ma il rendimento per flusso congelato (supponendo cioe che la romposizione del gas resti costante durante l'espansione, ed eguale a quella del ristagno), e molto basso rispetto all'elio che, essendo monoatomico, non si dissocia.

Per quanto riguarda le caratteristiche di ionizzazione e importante notare che un forte valore del potenziale di ionizzazione migliora il valore di $\varepsilon_{e l}$ al erescere di $I s p$. In tal caso, infatti, il grado di ionizzazione non varia eccessivamente al crescere della temperatura (e quindi della potenza dell'areo a pari portata) nella zona dell'arco e in conseguenza anche la conducibilita dell'areo non diminuise molto a il mpporto $R_{n} / R_{e}$ risulta solo poco minore.

La stabilita dell'arco deriva dalla dimensione dello stesso. Se l'arco ha una piccola scrione normale una accidentale distorsione di esso porta ad una resistenza maggiore e ad m pericolo di interruzione dell'arco (o comunque di instabilità); se la sezione normale è grande, come nel caso dei propellenti monoatomici, l'effetto e meno sentito. Per ovviare all'in- 
conveniente della instabilità, al forte flusso di calore alle pareti e, soprattutto, per migliorare il valore di $\varepsilon_{\text {tch }}$ si e proposto l'impiego di $H_{0}+L_{1}$; quest'ultimo elemento è monoatomico ed ha una bassa cenergia di ioniz-

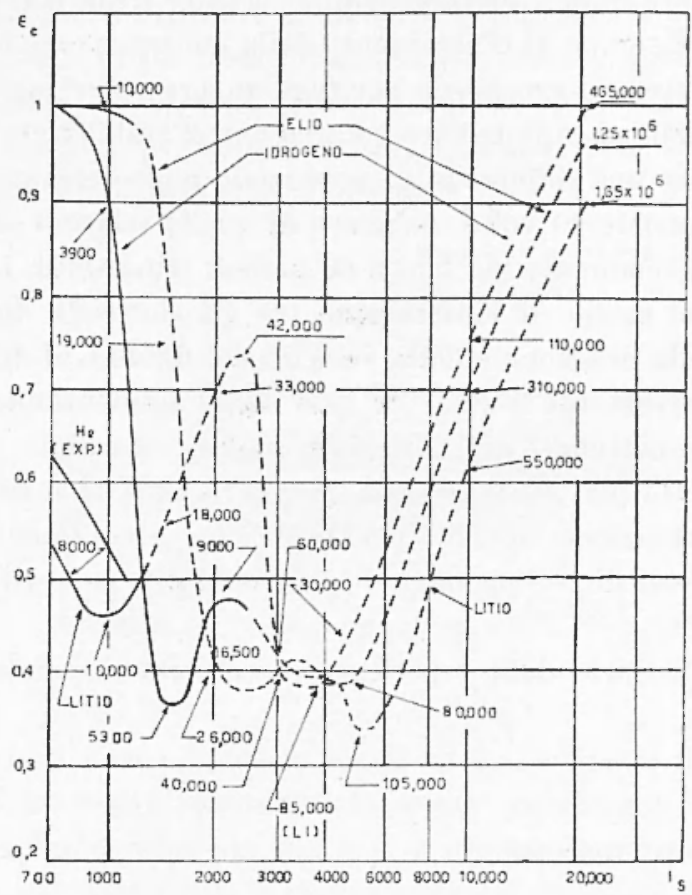

Fing. 5

zazione $(5,39 \mathrm{ev})$ rispetto all'idrogeno $(13,6 \mathrm{ev})$. Il $L$ e immesso nella zona dell'ar'o e l' $H_{2}$ forma un vortice attorno ad esso e protegge le pareti della camera. In tal modo puo aversi una temperatura della miscela abbastanza bassa all'ingresso dell'ugello e quindi un piceolo grrado di ionizzazione dell' $H_{\text {. }}$.

\section{7. - TIPO DI PO'TENZA ELE'I"IRICA.}

La scelta del tipo di corrente elettrica e abbastanza controversa il che è un indice che sia la corrente alternata che quella continua hamno un loro campo di applicazione; qualche volta il tipo adoperato dipende unicamente dal tipo di potenza elettrica disponibile. Isa corrente alternata ha il problema fondamentale della riacensione dell'arco due volte 
per ogni periodo; la facilita del ristabilimento dell'arco dipende sostanzialmente dalla conduttivita del fluido nella zona dell'areo rioe dal suo grado di ionizzazione all'atto dell'applicazione della diflerenza di potenziale. Orviamente questa ionizzazione, al cessale della col'rente, tende a diminuire e, se il tempo di rilassamento della ionizzazione ie breve rispetto al semiperiodo (tempo necessario per raggiungere valori alti della differenza di potenziale ai capi dell'areo) l'areo non si stabilizzerai pin, a meno che non si abbia nna differenza di potenziale molto elevata fino ad un valore del potenziale 20 volte maggione di quello relativo alle condizioni normali di funzionamento per c.a. a 60 periodi trifase) [2]. La velocita di diminuzione del grado di ionizzazione tra gli elettrodi diminuisce con l'aumentare della pressione e della velocita del fluido e al diminuire della temperatura circostante l'arco. Si puo agire ammentando il numero delle fasi e immettendo del materiale molto ionizzabile (secoling). La conente alternata puo essere comoda per la facilita della sua produzione e della trasformazione nonche per provocare spostamenti alternativi dell'areo, a mezzo di campi magnetici autoindotti, per evitare ablazioni sugli elettrodi.

La maggior parte delle esperienze e stata finora condotta con corrente continua.

\section{8. - AYVIAMENTO DELL'AR(O).}

11 problema dell'aviamento dell'areo presenta anche qualehe difficolta commessa con l'eventuale esigenza di dover proveredere a successive aceensioni. Un metodo ovvio e quello di portare a contatto i due elettrodi o commuque ad una distanza molto piccola; per fare cio si ha bisogno di un mecranismo di avanzamento, per esempio, del eatodo. Vero is che, a volte, un sistema di alimentazione può risultare necessario per alimentare il catodo a causa del consumo di esso durante il funzionamento; nel caso pero che questa alimentazione non sia prevista, un simile sistema potrebbe risultare troppo complesso. Si puo pensare ad un cireuito elettrico che funzioni da interruttore a mezzo di trasformatori ad impulsi che famo scoccare un piccolo areo in prossinita di due elettrodi e quindi innescano lareo principale; bisogna provvedere in tal caso an un isolamento di questo circuito o, meglio, ad un suceessivo allontanamento di essso dalla zona dell'arco per evitarne la distruzione. Il sistema più semplice e piu sicuro potrebbe essere quello di iniettare tra gli elettrodi un Huido conduttore oppure un gas a basso potenziale di ionizzazione. 
9. - RESISTO-JET.

Una menzione particolare merita un altro tipo di sistema elettrotermico: il resisto-jet $\left({ }^{7}\right)$. Come dalla stessa parola, questo è un sistema elettrotermico che sfrutta l'efietto Joule in una resistenza elettrica, di materiale speciale, per somministme energia, sotto forma di alore. ad un propellente a basso peso molecolatre (per es. $H_{2}$ o $H_{e}$ ).

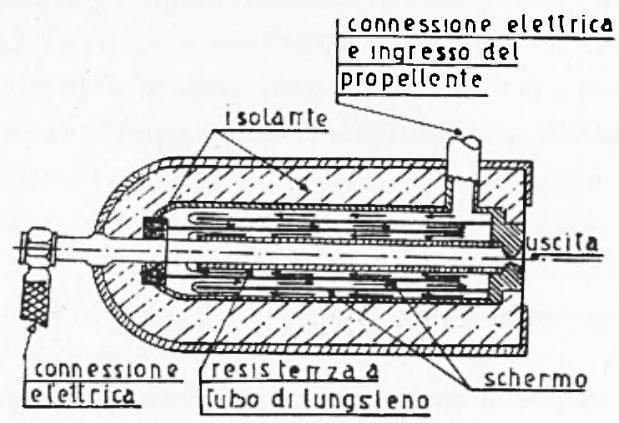

Fig. 6

Láa resistenza es di tungsteno a puo arrivare fino a temperature di $3000^{\circ}$ in atmosfera non ossidante; in queste condizioni con $H_{2}$ si puo' a rivare fino a $I s p=1000 \mathrm{sec}$. La Fig. 6 illustra il possibile schema di riscaldamento del propellente a rigenerazione. I vantaggi di questa soluzione sono:

1) semplicita e basso potenziale elettrico necessario per il funzionamento:

2) facilità della regolazione della spinta e dell'impulso specifico;

3) accoppiamenti a diversi generatori di corrente sia alternata che continua;

4) nessun problema di avviamento:

5) buoni valori di $\varepsilon_{\text {con }}$.

D'altra parte, pero, l'impiego e limitato a valori di $I s p \simeq 1000$ (per $H_{2}$ ) ed inoltre, per una durata ragionevole della resistemaa, non bisognal supelare il limite di $25 \mathrm{WV} / \mathrm{Am}^{2}$ di superficie.

L'impiego di materiali migliori del tungsteno (come carburi di Niobio, Tantalio, e Afnio) possono incrementare i valori di $I s p$ oltre il $10 \%$.

Per il resisto-jet la pressione operativa va scelta considerando che, da un lato, un valore alto di essa incrementa la trasmissione del calore 
dall'elemento resistivo al fluido e quindi incrementa il valore di $\varepsilon_{\text {con }}$, dall'altro incrementa le sollecitazioni e fa diventare critiche le dimensioni della sezione di gola a causa delle erosioni. In seguito a questo bilancio la pressione migliore sembra essere attorno a $2 \div 3$ atmosfere per impiego di $H_{2}$ e per una pressione all'esterno circa zero.

Infine resta il problema, che si vuole esaminare piu da vicino, del processo di conversione della energia del fluido a monte dell'ugello, in energia cinetica del getto all'uscita.

Il problema e comune a tutti i sistemi termici e pertanto l'importanza di realizzare buoni rendimenti di conversione va al di la dei sistemi elettrotermici anche se, per questi sistemi, date le altissime temperature di ristagno raggiungibili, il problema assume aspetti di particolare importanza.

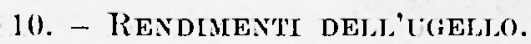

I'energia a disposizione per la conversione (entalpia totale del propellente) e immagazzinata in diverse forme energetiche associate ai gradi di liberta interna (traslatori, rotatori, vibratori) e ai fenomeni di dissociazione e di ionizzazione delle particelle.

Questi gradi di liberta sono eccitati, cioe esiste una certa energia ad essi associata, man mano che la temperatura cresce.

Poiche esiste la possibilità di scambio tra le forme energetiche sopraelencate, via via che l'energia traslatoria viene convertita nell'ugello in energia cinetica, gli altri gradi di liberta, per ristabilire le condizioni di equilibrio che comportano una ben definita distribuzione dell'energia totale fra tutti i gradi di liberta, cedono parte della energia ad essi associata al grado di liberta traslatoria.

Questi scambi sono caratterizzati, da un certo numero statistico di collisioni e, poiche l'intervallo medio del tempo tra due collisioni successive e finito ed e determinato dal valore della pressione e della temperatura, si avra un valore finito del tempo necessario per il raggiungimento dell'equilibrio (tempo di rilassamento e tempo rhimico caratteristico, se si parla di equilibrio chimico tra diverse speci molecolari).

Il raggiungimento dell'equilibrio di un particolare processo, come si mettera meglio in risalto in seguito, nel moto di un fluido si ha solo se il tempo caratteristico del processo e trascurabile rispetto ad un tempo caratteristico macroseopico (nel caso particolare rappresentato, per esempio, dal tempo di permanenza della particella fluida nell'interno dell'u- 
gello). Si puo anticipare che, per espansioni forti e ugelli corti, dati i brevissimi tempi di permanenza, difficimente si raggiungeramo le condizioni di equilibrio e quindi parte dell'energia, che in equilibrio sarebbe disponibile per la conversione in energia cinetiea, e invece escrusa dal processo di conversione e resta contenuta nel fluido allo scarico.

Un'analisi tei tempi caratteristici ha mostrato che gli scambi energetici tra i gradi di liberta traslazionali, rotazionali e vibrazionali avvengono in tempi molto piccoli rispetto al tempo macroscopico $t_{a r}$ (anche se il tempo di rilassamento vibrazionale e piu lungo di quello rotazionale di diversi ordini di grandezza) e poiche, viceversa, i tempi caratteristici chimici delle reazioni di dissociazione e ionizzazione sono relativamente lunghi rispetto a $t_{M}$ (per ugelli (onvenzionali e forti espansioni), si puo definire flusso congelato quello per il quale la composizione chimica resta costante e uguale a quella all'ingresso dell'ugello mentre i gradi di liberta traslatoria, rotatoria e vibratoria (energia termica) sono in equilibrio.

Il mancato raggiungimento dell'equilibrio in un ugello di scarico puo essere valutato globalmente, ai fini propulsivi, a mezzo di un rendimento, che si indica con $\varepsilon_{t c h}\left(^{*}\right)$, che esprime il rapporto tra l'energia convertita in energia cinetioa $\left(h_{i}-h_{u}\right)$ e l'energia che sarebbe convertita se il fluido fosse in condizioni di equilibrio all'interno dell'ugello $\left(h_{t}-h_{u}^{c}\right)$.

$$
\varepsilon_{\mathrm{tch}}=\frac{h_{i}-h_{u}}{h_{i}-h_{\mathrm{t}}^{e}}
$$

dove gli indici $i$ ed $u$ indicano, rispettivamente, le condizioni all'ingresso e all'uscita dell'ugello di scarico.

Il rendimento $\varepsilon_{\text {teh }}$, come definito dalla [5] potrat variare entro i limiti $1 \leqslant \varepsilon_{\text {tch }} \leqslant \varepsilon_{c}$ dove

$$
\varepsilon_{c}=\frac{h_{i}-h_{u}^{c}}{h_{l}-h_{u}^{e}}
$$

e il rendimento congelato e $h_{u}^{c}$ e la entalpia competente al gas all'uscita dell'ugello se la composizione rimanesse costante ed uguale a quella del gas all'ingresso dell'ugrello (efflusso congelato). Evidentemente $h_{w}^{c}$ e il valore massimo della entalpia alio scarico che puo aversi con una certa geometria, con certe condizioni iniziali e con un certo valore dela pressione esterna; a questo valore corrisponde il minimo valore del rendimento di non/equilibrio $\varepsilon_{\text {tch }}$.

(*) L'indice tch vuole indicare che tale rendimento dipende da un tempo chimico caratteristico diverso da zero. 
Viceversa, se i tempi caratteristici chimici fossero molto minori del tempo caratteristico macroscopico, al limite se i loro rapporti sono zero, si potrebbero realizzare, durante la fase di espansione, le condizioni di equilibrio chimico e quindi arere la massima energia possibile convertita in energia cinetica $\left(h_{u}=h_{u}^{e} ; \varepsilon_{\mathrm{tch}} \equiv 1\right)$.

In pratica si avra un valore di $\varepsilon_{\text {tch }}<1$ e quindi non tutta l'energia immagazzinata sotto forma chimica (normalmente dissociazione) verra recuperata nell'ugello.

Il rendimento adiabatico dell'ugello si definisce:

$$
\varepsilon_{\mathrm{ad}}=\frac{h_{i}-h_{u}^{e}}{h_{i}}
$$

cioe esso e il lapporto tra l'energia cinetica all'uscita, per un processo di espansione in equilibrio termodinamico, a l'energia contenuta nel fluido all'ingresso dell'ugello. In assenza di cause dissipative all'interno del gas e per espansione adiabatica:

$$
\varepsilon_{\mathrm{ad}}=f\left(A^{*} / A_{u}, P^{*} / P_{u}\right)
$$

dove $A^{*}$ e $p^{*}$ sono, rispettivamente, i valori critici dell'area della sezione normale e della pressione (*). Evidentemente il rendimento globale dell'ugello è $\varepsilon=\varepsilon_{\text {tch }} \varepsilon_{\mathrm{ad}}$.

Il considerare, accanto a $\varepsilon_{\text {ad }}$ anche il rendimento $\varepsilon_{\text {tch }}$ è il fatto nuovo della propulsione elettrotermica rispetto alla chimica; infatti, se prima si poteva trascurare un non equilibrio chimico durante l'espansione perche esso portava a perdite di non pii del $5 \div 10 \%$ dell'energia utilizzabile, adesso, invece, date le temperature raggiunte e dato che i livelli energetici sono superiori ai livelli di dissociazione e, spesso, anche a quelli di ionizzazione, queste perdite possono raggiungere valori molti alti, per es. nel caso di $H_{2}$ e $I s p \simeq 1500 \mathrm{sec}$ (V. Fig. 5) si ha $\varepsilon_{c}=0,35$. Questo e un valore quasi inacectabile e pertanto nasce il problema di come migliorare l'equilibrio nella fase di espansione in modo tale che $\varepsilon_{\text {tch }}$ risulti sostanzialmente maggiore di $\varepsilon_{c}$.

Si puo pensare di utilizzare propellenti che, a parita di $I s p$, esibiscano valori piu elevati di $\varepsilon_{c}$ in modo tale da non doversi stare a preoceupare eccessivamente del raggiungimento dell'equilibrio nell'ugello. Questo e

$\left({ }^{*}\right)$ Nel caso piu semplice in cui $c_{p}=$ cost. ed espansione corretta $\left(p_{u}=p_{\text {est }}\right)$ si ha: $\varepsilon_{\mathrm{ad}}=1-\left(\frac{p_{u}}{p_{i}}\right)^{\frac{\gamma-1}{\gamma}}$. 
il caso di propellenti monoatomici per i quali, ovviamente, son si ha il fenomeno della dissociazione (per es., per $H_{e}$ a $I s p \simeq 1300, \varepsilon_{c} \simeq 0,95$ [Tedi Fig. 5]]).

D'altro canto pero, se si pensa a sistemi propulsivi ad elevatissimo Isp dovra adoperarsi necessaliamente l'idrogeno che ha il piu basso peso atomico e quindi raggiungera il massimo $I s p$ a parita di temperatura totale rispetto a qualsiasi altro propellente. Eceo quindi la necessita di studiare la fase di espansione allo scopo precipuo di migliorarne il valore di $\varepsilon_{\text {tch. }}$. Questo e stato fatto, in una analisi approssimata in $\left({ }^{10}\right)$. partendo dalle equazioni dell'aerotermochimica, linearizzandole e discutendo alemi casi di maggiore interesse pratico. Si sono individuati i parametri essenziali che entrano in gioco nel processo e si e pervenuto a dei criteri per la determinazione del profilo dell'ugello onde migliorare il valore di $\varepsilon_{\text {ten. }}$.

\section{1. - Proposte.}

In relazione alla disamina fatta si sono considerate alcune possibilità per massimizzare il valore di $\varepsilon_{\text {teh }}$.

Le proposte cui qui si accenna, sono attualmente in esame presso l'Istituto d'Aerodinamica dell'Universita di Napoli e, tramne quando se ne fa esplicitamente menzione, non sembra siano state esaminate da altri autori.

\section{1. - Ugelli non convenzionali.}

Per rendere il grado di dissociazione minimo si puo pensare di fare ricorso ad ugelli non convenzionali che sostituiscono la espansione graduale, all'intemo di un ugello convenzionale, con una espansione attorno ad uno spigolo (ugello a spina) (Vedi Fig. 7). Per questi ugelli (come anche per ugelli a espansione-deflessione) la corrente, in corrispondenza della sezione di gola, non è diretta lungo l'asse di simmetria dell'ugello (direzione della spinta). Nell'ugello a spina essa e diretta verso l'interno ed il rapporto tra la pressione critica e quella ambiente e tale da far espandere la corrente attorno allo spigolo esterno in modo che alla fine dell'espansione la corrente e diretta assialmente. La parte interna subisce, invece, una variazione graduale di direzione fino a portarsi anche essa in direzione assiale. 
I vantaggi di questa soluzione, gia proposta per razzi chimici convenzionali in assenza di fenomeni di non equilibrio, sono:

1) riduzione della lunghezza (circa il $40 \%$ di un ugello convenzionale);

2) impossibilità di sovla-espansione, e quindi assenza di onde d'urto, al variare della pressione esterna;

3) semplificazione del problema del raffreddamento.

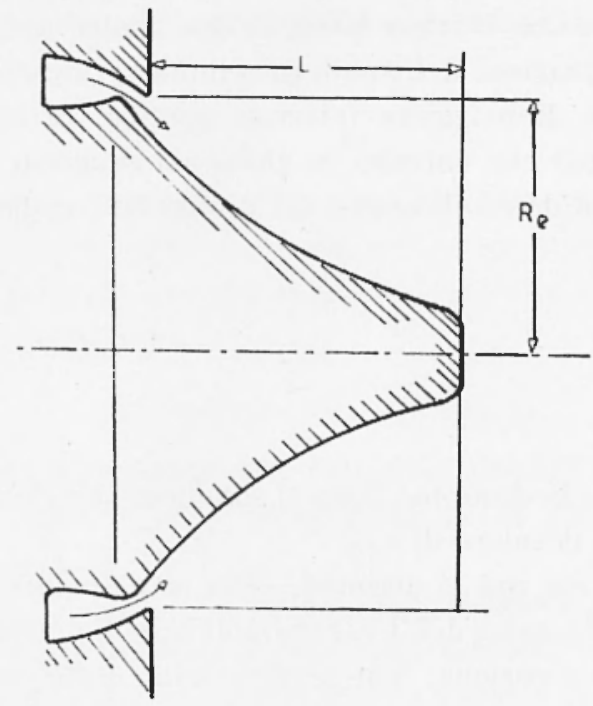

Fig. 7

Un simile ugello può risultare particolarmente interessante, per il ampo in esame, cioe in presenza di fenomeni di non-equilibrio, data la rapida espansione iniziale, con il conseguente grande volume occupato dal gas durante il processo di espansione $\left({ }^{10}\right)$, e l'assenza di sovra-espansione. Andrebbero comunque fatti studi teorici e determinazioni sperimentali al riguardo onde controllarne i vantaggri.

\section{2. - Ugelli ad espansione incorretta (*).}

Un'espansione incolretta, in presenza di condizioni di non equilibrio, risulta notevolmente complessa per la eventuale presenza di discontinuità termofluidodinamiche e chimiche.

$\left(^{*}\right)$ Si intende per espansione incorretta quella per la quale la pressione all'uscita dell'ugello $\left(p_{u}\right)$ e diversa dalla pressione ambiente $\left(p_{a}\right)$. 
Riferendosi ad una stessa lunghezza dell'ugello e ad una espansione corretta, ma sovra-espansione, che peggiora il valore di $\varepsilon_{a d}$ ha un effetto complesso su $\varepsilon_{\text {tch }}$; infatti valori piu bassi della pressione, da un lato incrementano il valore del volume a disposizione (a pari lumghezze), dall'altro, pero, provocano una diminuzione di $\frac{T_{\mathrm{i}} P_{\alpha}}{V_{0}}$ e quindi possono in definitiva provocare sia un aumento che una diminuzione di $\varepsilon_{\mathrm{tcl}}\left({ }^{10}\right)$.

L'effetto di una sotto-espansione, invece porterebbe, a prima vista, ad una diminuzione di $\varepsilon_{\text {ten }}$ perche il propellente si trovera alluscita dell'ugello con grado di dissoriazione (o ionizzazione) maggiore di quello che avrebbe se fosse alla pressione (minore) esterna.

\section{3. - Iniezione Catalitica.}

Si puo pensare di incrementare $L_{1} B_{a}$ o, globalmente, i parametri temodinamici da cui dipende il grado di dissociazione allo scarico $\left({ }^{10}\right)$, che sono funzioni di stato, iniettando, per esempio, un opportmo fluido

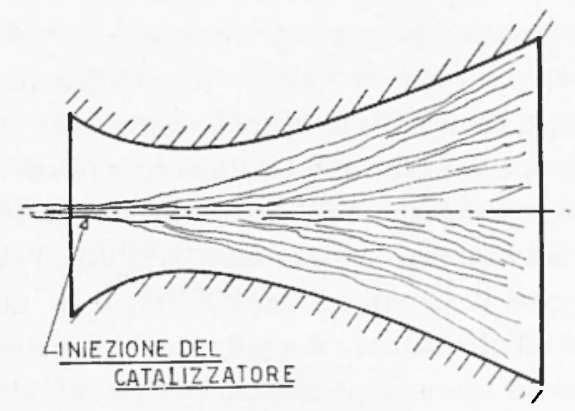

Fig. 8

catalizzatore per la reazione di riassociazione (Vedi Fig. 8) e di neutralizzazione. Le cantteristiche di tale fluido dovamo essere quindi tali da incrementare la stabilità chimica del sistema (cioe ammentare la forza, o affinità che tende a richiamare il sistema nelle condizioni di equilibrio). Un problema analogo e stato, molto recentemente, indagato (11) prendendo in considerazioni superfici catalitiche per la ricombinazione.

\section{1.t. - Sistemi a piu reazioni.}

Si puo pensare di realizzare un opportuno accoppiamento con una o piu reazioni chimiche la cui presenza influenzi, nel senso roluto, la velocita della reazione principale (riassociazione o neutralizzazione). Tel caso 
di due reazioni le equazioni cinetiche dovranno seriversi, nei limiti dell'approssimazione lineare della termodinamica dei processi irreversibili:

$$
\begin{aligned}
& a_{1}=-L_{11} A_{1}-L_{12} A_{2} \\
& a_{2}=-L_{21} A_{1}-L_{22} A_{2}
\end{aligned}
$$

dove $\alpha_{i}$ sono le velocita di reazioni chimiche (per la reazione principale sara la velocita di dissociazione), $A_{i}$ sono le affinita chimiche e $L_{i j}$ sono $\mathrm{i}$ coefficienti cinetici.

I parametri adimensionali delle analisi avranno una forma pii complessa, di quelli di cui in $\left({ }^{10}\right)$, per la presenza tra l'altro, del coefficiente cinetico $L_{12}$ con il segno +0 - a seconda dell'accoppiamento tra le reazioni. Una prima indagine preliminare $\left({ }^{12}\right)$ e stata fatta per il caso di due reazioni chimiche, e si sono determinate il segno delle variazioni delle lunghezze di rilassamento in dipendenza del segno dell'accoppiamento delle reazioni (segno di $L_{12}=L_{21}$ ).

L'importanza di questi risultati e la loro applicabilita puo comprendersi quando si pensa alla possibilita di fare avvenire, nell'ugello, una reazione artificiale creata con la introduzione di una o piu sostanze.

Il caso gia analizzato teoricamente e relativo ad un condotto a sezione costante all'ingresso dal quale il fluido e in condizione di non equilibrio. Il caso dell'ugello, pur non permettendo le immediate deduzioni fatte nel caso di area costante, e analogo; infatti dopo una prima rapida espansione la miscela non sara in condizione di equilibrio; partendo da una data condizione, bisogna "sovrapporre" i due effetti di una velocita di reazione, che tende a riportare il sistema in equilibrio, e di una divergenza del condotto che tende ad allontanare maggiomente il sistema dall'equilibrio. Il sistema si presta ad essere linearizzato per piccole divergenze del condotto e la soluzione di ordine zero e proprio quella ad area costante gia discussa. E evidente che per pratiche applicazioni, il rapporto tra la massa dei reagenti della reazione "principale" (cioe quella relativa al processo di conversione in energia traslazionale delle altre forme di energia del propellente) e quelle della reazione "pilota " (cioe quella artificialmente provocata) deve essere il maggiore possibile. Cioe, in sostanza, dovrebbe essere minima la quantita di additivi che si immettono nel flusso (potrebbe, per esempio, bastare una sola sostanza che reagisce con il propellente usato e che, con tale reazione, acceleri la reazione di riassociazione o di neutralizzazione).

\section{5. - Immissione di energia nell'ugello.}

Un ultimo criterio puo essere quello di rendere minimo il grado di dissociazione o ionizzazione del propellente. Si puo pensare che realizzare 
aio limitando non la temperatura totale, determinata dal valore dell'energia immessa e dall'Isp che si vuole realizzare, ma la temperatura statica, questo si puo fare addizionando energia al propellente mentre questo si espande e quindi tende a raffreddarsi (*).

it ben evidente che questo porta ad una diminuzione di $\varepsilon_{a d}$ (l'energia viene somministrata ('on grado di sfruttabilita minore) ma, se si realizza un sensibile migliommento del valore di $\varepsilon_{\text {tch }}$ (al limite 1 , se non si oltrepassano i livelli energetici della dissociazione e ionizzazione), può clarsi si abbia un valore massimo del prodotto $\varepsilon_{\text {tch }} \times \varepsilon_{a d}$ e ne risulti, pertanto, una convenienza nel seguire questo ariterio.

\section{2. - Conclusioni.}

Si sono indicati i problemi relativi alla costruzione e alle applicazioni di sistemi propulsivi elettrotermici ed in particolare si e esaminato il comportamento dell'ugello. Il problema e comune a quello degli ugelli di razzi termici spinti per i quali cioe si superano i livelli energetici di dissociazione del propellente adoperato. Riferendosi ad una analisi linearizzata, che ha messo in luce i parametri che direttamente influenzano la conversione di energia chimica in energia cinetica in un ugello in presenza di fenomeni di non equilibrio, si sono accemnate ad alcune proposte, da indagare ulteriormente, allo scopo di migliorare il rendimento $\varepsilon_{\text {tch }}$ che penalizza sempre maggiomente i sistemi termici all'aumentare dell'impulso specifico.

\section{BIBIIOGRAFIA}

(1) MaC C. Adans and Iortox Camac, The Are Ileated Plasma Thrust Chamber. Presented at the ARS Controllable Satellites Conference, II.I.'T., April 30-IIay 1, 1959 (791-59).

$\left({ }^{2}\right)$ J. W. Reid, Recent Advances in Ihigh Pressure, High Power Ares, American Rocket Society - Space Flight Report to the Nation. New York Coliseum, October 9-15, 1961 (2127-61).

(3) R. R. Joms, I. F. Coxvors, A. Mrroner and II. Macomber, Are Jet Engine Performance - Experiment and Theory. Presented at the ARS 15th Annual Neeting, shoreham Ilotel, Washington, D.C., December $5-9,1960(1505-60)$.

(*) Bisogna tenere pero presenti le ovvie complicazioni tecnologiche connesse con il sistema di inmissione di energia in una corrente fluida ed entalpia totale molto elevata. 
(1) H. O. Noeske, R. R. Kassier, Analylical Investigation of A Bipropellent Arcjet. American Rocket Society space Flight Report to the Nation, New York Coliseum, October 9-15, 1961 (2125-61).

$\left({ }^{5}\right)$ Tugnas R. Brociax, Preliminary Evaluation of IIelium as an Electric Arc Ireated Propellant. Presented at the ARS 13th Annual Meeting, New York, N.Y. Nov., 17.21, 1958.

(8) Michane I. Yarmovycil, Fraxcis J. Davidson, JaCk Lambert, Arax Mroner, The Are Jet Propelled Space Tehicle. American Rocket Society space Flight Report to the Nation. New York Coliseum, Oetober 9-15, 1961 (2131-61).

(7) Janes II. Howard, The Resistojet. American Rocket Society Space Flight Report to the Nation; New York Coliseum, October 9-15, 1961 (2126-61).

(8) Gorbon I. Canj Adriano, C. Ducati, Gabriet II., Giannini Willis d. Stoney, The tre jet. Second ddvances Propulsion Symposium, Cambridre Massachussetts on $7-8$ October 1959.

(') M. CAMAC and G. S. JANEs, Applied Magnetohydrodynamics at itvoo Everelt Research Laboratory. Presented at the ARS-Northwestern Gas Dynanies Symposium, Northwestern Iniversity, Evanston, Illinois, Lugust 24-26, 1959 (902-59).

$\left.{ }^{(10}\right)$ R. Mosrr, Analisi linearizzata sull'eflusso non in equilibrio in ugelli di razzi. In corso di pubblicazione.

(11) S. S. Pexier and J. Ponter, Chemical reactions during in Rocket nozzles. AROD 834: 52, Tech. Report 37. Febr. 1962.

(12) L. G. Napolitaxo, Moti linearizati non in equilibrio di miscele bireagenti. Atti del simposio sulle Alte temperature, Politecnico di Torino, 9-12 sett. 1962. 